\title{
Flux crystal growth and the electronic structure of $\mathrm{BaFe}_{12} \mathrm{O}_{19}$ hexaferrite
}

\author{
V.V. Atuchin ${ }^{1,2,3}$, D.A. Vinnik ${ }^{4}$, T.A. Gavrilova ${ }^{5}$, S.A. Gudkova ${ }^{4,6}$, L.I. Isaenko ${ }^{3,7}$, Xingxing

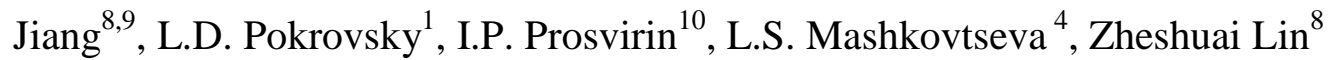

${ }^{1}$ Laboratory of Optical Materials and Structures, Institute of Semiconductor Physics, SB RAS, Novosibirsk 630090, Russia

${ }^{2}$ Functional Electronics Laboratory, Tomsk State University, Tomsk 634050, Russia

${ }^{3}$ Laboratory of Semiconductor and Dielectric Materials, Novosibirsk State University, Novosibirsk 630090, Russia

${ }^{4}$ South Ural State University, 76 Lenin Aven, Chelyabinsk 454080, Russia

${ }^{5}$ Laboratory of Nanodiagnostics and Nanolithography, Institute of Semiconductor Physics, SB RAS, Novosibirsk 630090, Russia

${ }^{6}$ Moscow Institute of Physics and Technology (State University), 9 Institutskiy Per., Dolgoprudny 141700, Russia

${ }^{7}$ Laboratory of Crystal Growth, Institute of Geology and Mineralogy, SB RAS, Koptyug Aven, Novosibirsk 630090, Russia

${ }^{8}$ BCCRD, Key Laboratory of Functional Crystals and Laser Technology, Technical Institute of Physics and Chemistry, Chinese Academy of Sciences, Beijing 100190, China ${ }^{9}$ University of Chinese Academy of Sciences, Beijing 100049, China

${ }^{10}$ Boreskov Institute of Catalysis, SB RAS, Novosibirsk 630090, Russia 
Table 1S. $d$-spacing values observed in TEM pattern shown in Fig. 1S

\begin{tabular}{|c|c|c|c|c|c|c|c|c|c|c|}
\hline$d_{\text {exp. }}(\AA)$ & 5.3 & 4.7 & 4.26 & 2.92 & - & 2.65 & 2.54 & 2.48 & 2.17 & 2.14 \\
\hline$d_{\text {tabl }}(\AA)$ & 4.98 & 4.67 & - & $\begin{array}{c}2.95 \\
2.9\end{array}$ & 2.78 & 2,63 & 2.55 & 2.42 & 2.24 & 2.13 \\
\hline $\begin{array}{c}\text { Intensity } \\
\text { rel. }\end{array}$ & 8 & 12 & - & $45 \quad 15$ & 89 & 100 & 9 & 47 & 30 & 19 \\
\hline$h k l$ & 101 & 102 & - & 110008 & 107 & 114 & 200 & 203 & 205 & 206 \\
\hline
\end{tabular}




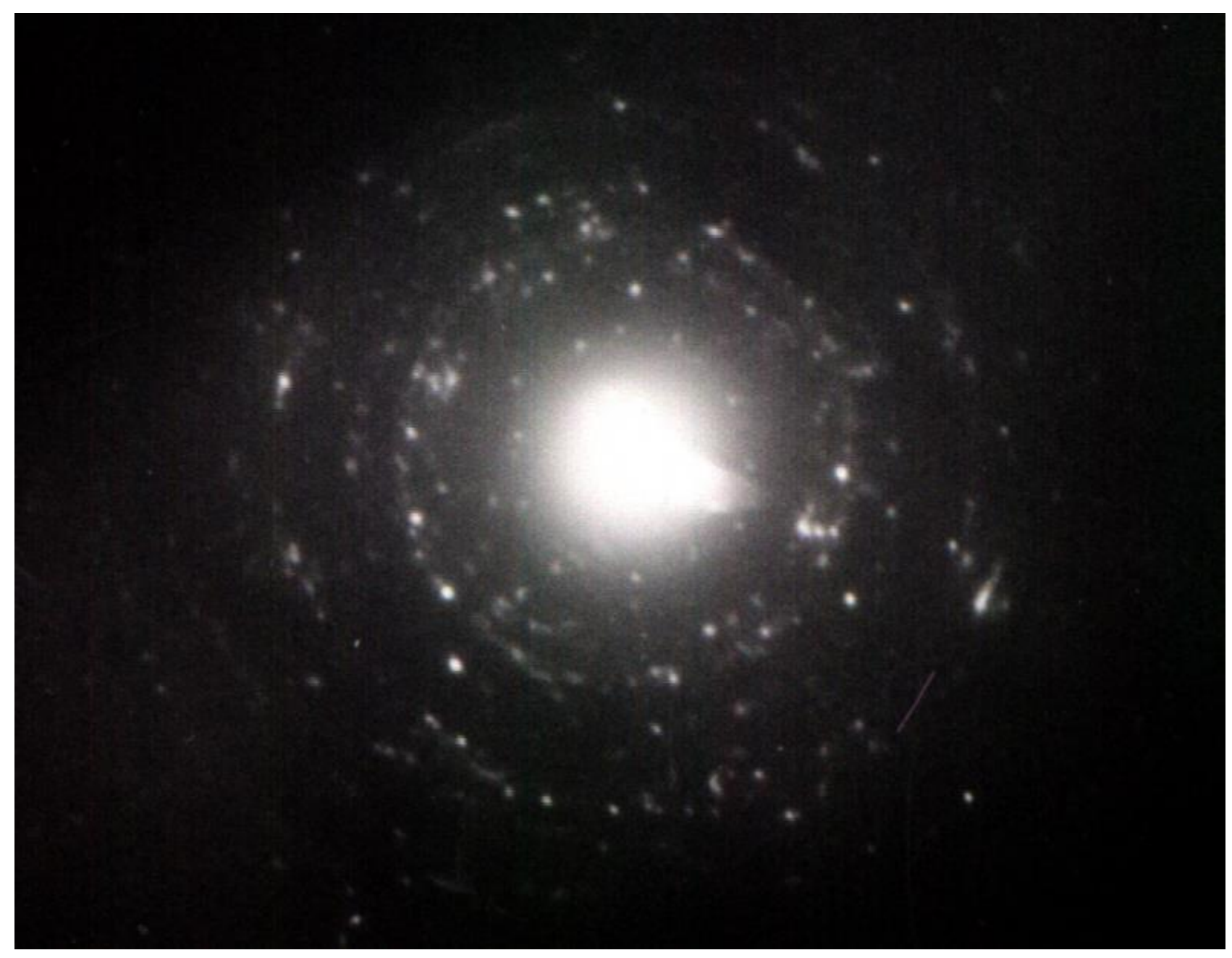

Fig. S1. TEM pattern typically observed from the $\mathrm{BaFe}_{12} \mathrm{O}_{19}$ sample. 


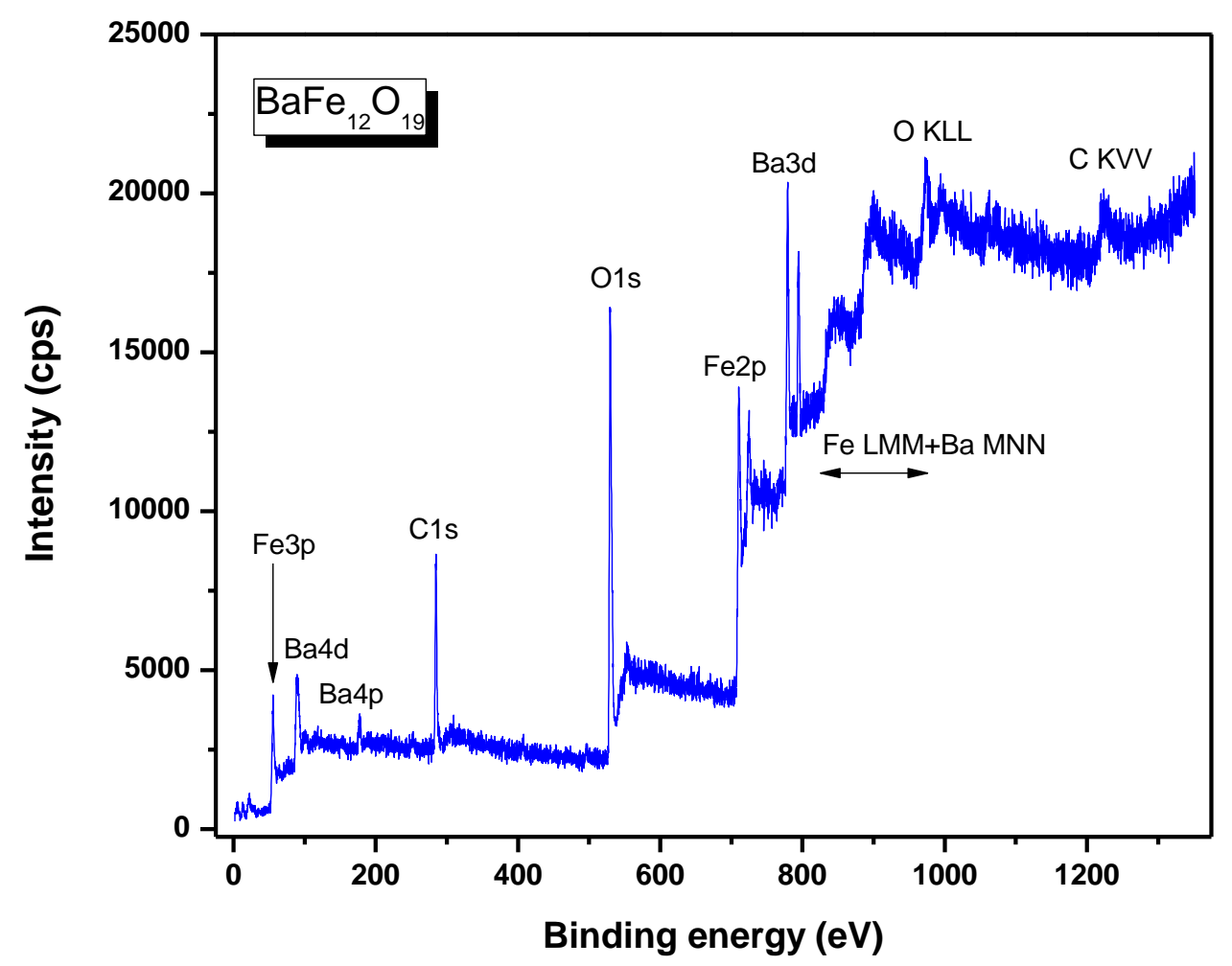

Fig. S2. Survey XPS spectrum of the $\mathrm{BaFe}_{12} \mathrm{O}_{19}$ powder. 


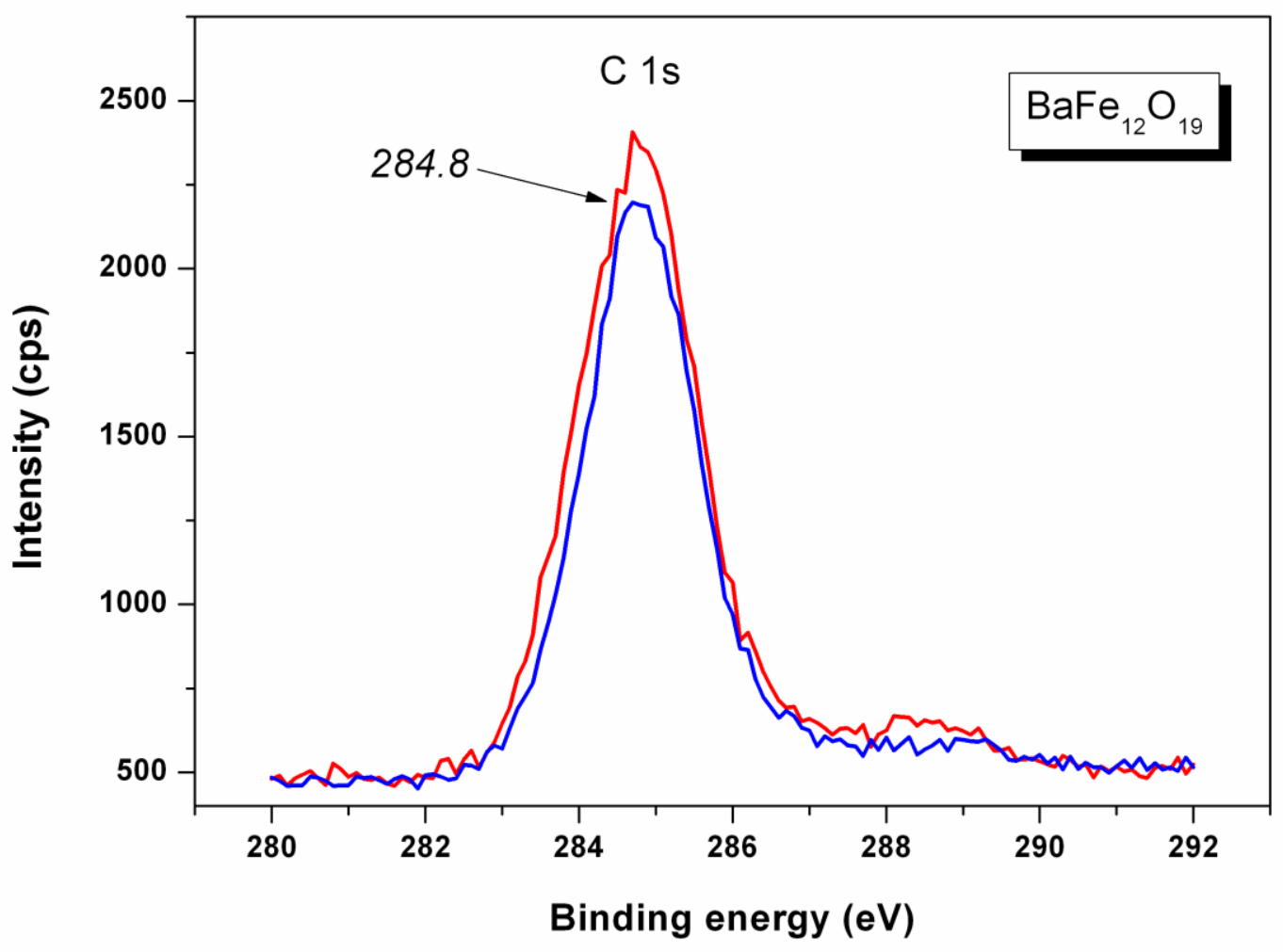

Fig. S3. The detailed spectra of C 1s core level recorded from initial (red) and bombarded (blue) sample. 


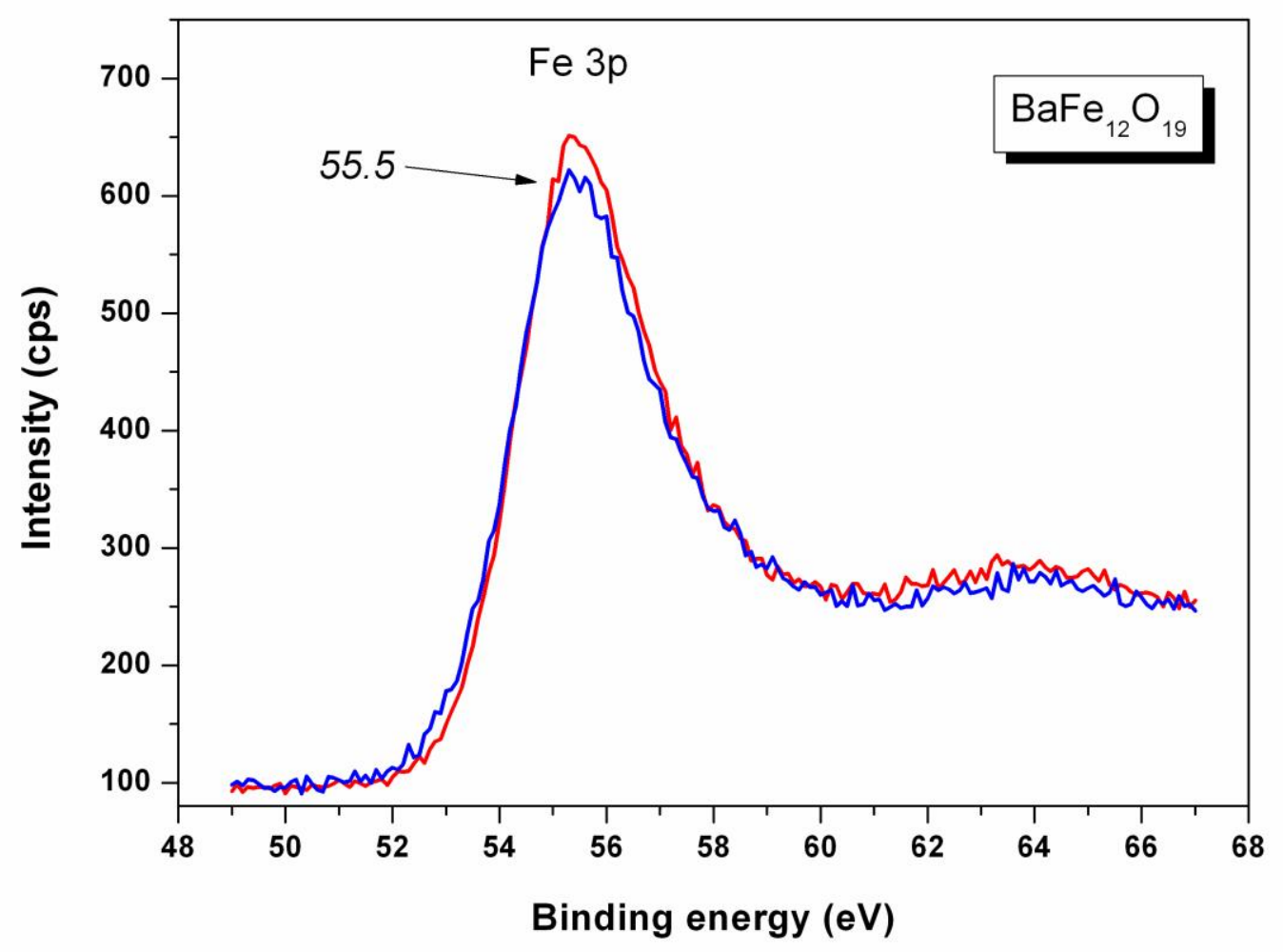

Fig. S4. The detailed spectra of $\mathrm{Fe} 3 \mathrm{p}$ core level recorded from initial (red) and bombarded (blue) sample. 


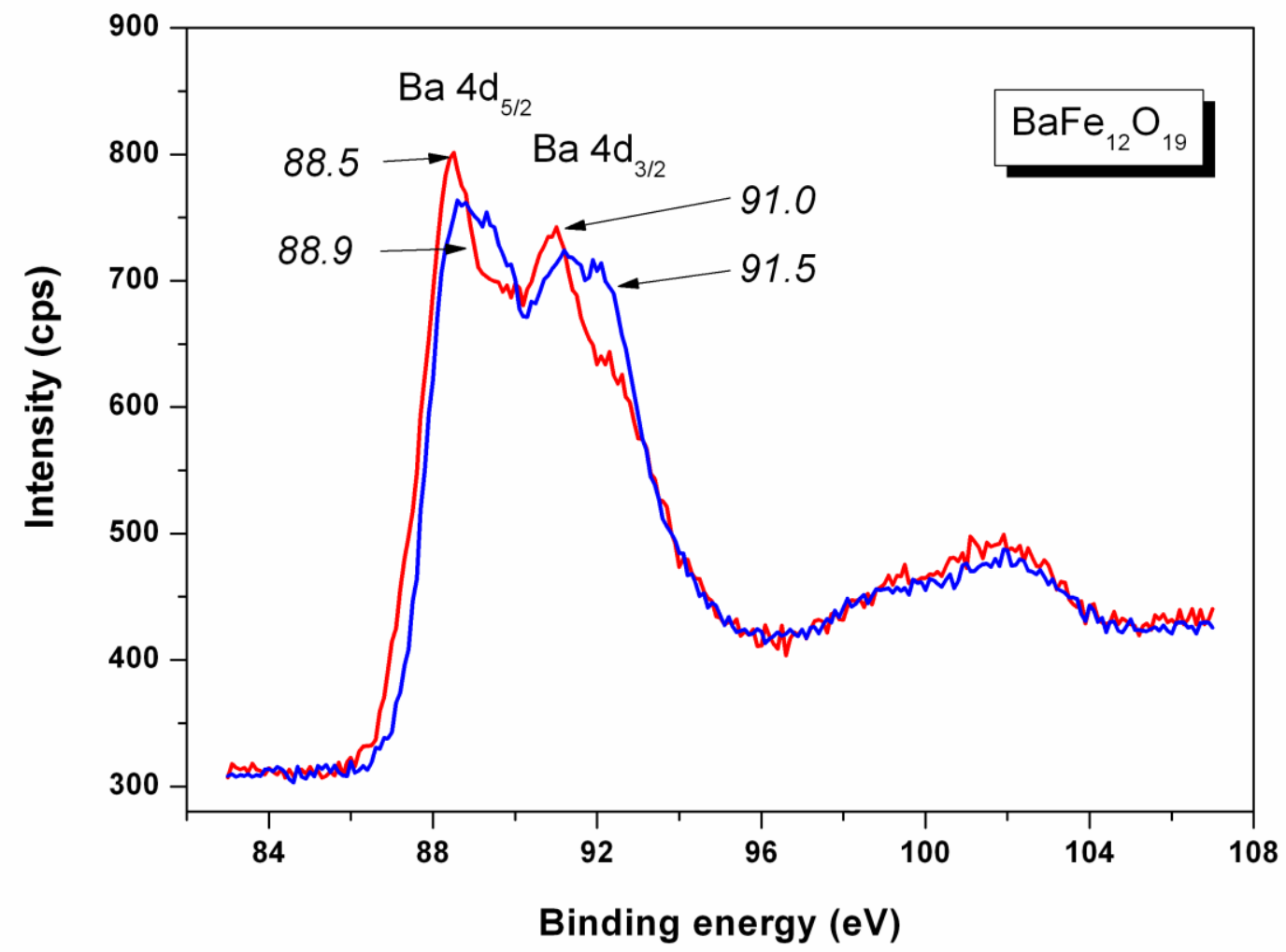

Fig. S5. The detailed spectra of $\mathrm{Ba} 4 \mathrm{~d}$ doublet recorded from initial (red) and bombarded (blue) sample. 